\title{
ESTUDO DO COMPORTAMENTO DA CREATINO QUINASE (CK) E CREATINO QUINASE-MB (CK-MB) SÉRICA DE CÃES SUBMETIDOS À OXIGENAÇÃO POR MEMBRANA EXTRACORPÓREA (ECMO) DURANTE UM PERÍODO DE TRÊS HORAS
}

\author{
Felipp Silveira Ferreira, ${ }^{1}$ Lara Lages Silveira, ${ }^{1}$ Alessandra Castello Costa, ${ }^{1}$ Antonio Peixoto Albernaz, ${ }^{2}$ \\ Claudio Baptista Carvalho ${ }^{3}$ e André Lacerda Abreu Oliveira ${ }^{2}$
}

1. Doutorando em Ciência Animal, Universidade Estadual do Norte Fluminense Darcy Ribeiro, Brasil. E-mail: felipp@uenf.br

2. Professor associado da Universidade Estadual do Norte Fluminense Darcy Ribeiro, Brasil

3. Professor titular da Universidade Estadual do Norte Fluminense Darcy Ribeiro, Brasil.

RESUMO

A oxigenação extracorpórea por membrana (ECMO) é uma técnica de suporte cardiopulmonar prolongado, que objetiva auxiliar os pulmões e/ou o coração quando estes apresentam processos de falência não responsiva aos tratamentos convencionais. Por não se tratar de um procedimento fisiológico, representa um grande desafio para a medicina, que busca torná-la um procedimento mais seguro. Dessa forma, procedeu-se a esta pesquisa objetivando avaliar o comportamento dos marcadores cardíacos $\mathrm{CK}$ e CK-MB de cinco cães submetidos à ECMO durante três horas. Empregaram-se cães sem raça definida, de diferentes idades, peso e sexo. Sob manutenção anestésica, os animais foram submetidos à
ECMO por canulação femoral, em um desvio arteriovenoso (AV). Estabelecido o circuito, mensuraram-se as variáveis a cada trinta minutos, durante um período de três horas. Os dados foram analisados estatisticamente com os testes de Anova, Tukey e Correlação de Pearson, com $\alpha=5 \%$. Os resultados apontaram um aumento sérico da CK e da CK-MB, caracterizando uma lesão muscular ao longo do procedimento. Os resultados apresentados apontam que a ECMO induziu uma lesão muscular não cardíaca por mecanismos fisiológicos. Conclui-se que a ECMO é uma técnica de suporte viável e que não induz lesões miocárdicas em cães durante um período de três horas.

PALAVRAS-CHAVES: CK, CK-MB, cães, ECMO, fisiologia.

\section{ABSTRACT}

\section{STUDY OF THE BEHAVIOR OF SERIC CREATINE KINASE (CK) AND CREATINE KINASE-MB (CK-MB) OF DOGS SUBMITTED TO EXTRACORPOREAL MEMBRANE OXIGENATION (ECMO) FOR THREE HOURS}

The extracorporeal membrane oxygenation (ECMO) is a technique of prolonged cardiopulmonary support, which aims to help the lungs or the heart when these organs present failure processes not responsive to conventional treatments. As it is not a physiological procedure, it represents a major challenge for medicine, which seeks to make it a safer procedure. Thus, this research was carried out to determine the course of the cardiac markers CK and CK-MB of five dogs submitted at ECMO for three hours. Mongrel dogs of various ages, weight and sex were used. Under anesthesic maintenance, the animals were subjected to femoral cannulation for ECMO, in an arterial-venous (AV) deviation. Once the circuit was established, the variables were measured every thirty minutes for a period of three hours. The data were statistically analyzed with Anova, Tukey and Pearson Correlation, with $\alpha=5 \%$. The results showed an increase of serum $\mathrm{CK}$ and $\mathrm{CK}-\mathrm{MB}$, characterizing a muscular injury during the procedure. The results showed that ECMO induced a cardiac muscle injury by a physiological mechanism. It was concluded that ECMO is a viable technical support and do not induce myocardial injury in dogs during a period of three hours.

KEYWORDS: CK, CK-MB, dogs, ECMO, physiology. 


\section{INTRODUÇÃO}

A oxigenação extracorpórea por membrana - ECMO -, derivada de extracorporeal membrane oxygenation, é uma técnica de suporte cardiopulmonar prolongado, empregada para auxiliar o pulmão e/ ou o coração, especialmente de neonatos e crianças, quando estes apresentam processos de falência não responsiva aos tratamentos convencionais não invasivos (BARTLETT et al., 1974; WEBER et al., 1990; ZWISCHENBERGER et al., 2000; THAKAR et al., 2001; MOSCARDINI et al., 2002; THAKAR et al., 2002; LIN et al., 2006; PEEK et al., 2006; TAMESUE et al., 2006; KAHN et al., 2007). Essa assistência respiratória extracorpórea prolongada deve ser aplicada a um grupo específico de pacientes em que a insuficiência respiratória for causada por uma enfermidade potencialmente reversível (SOUZA \& ELIAS, 2006).

Por se tratar de um procedimento altamente invasivo, é certo que a circulação extracorpórea determina alterações no sangue e nos tecidos perfundidos, gerando um quadro geral de inflamação ou autoagressão. Inicialmente reconhecida com denominações tais como síndrome pós-perfusão e/ou pulmão de bomba, passou, posteriormente, a ser conceituada como reação inflamatória geral do organismo. As manifestações clínicas se caracterizam por alterações funcionais muito ou pouco intensas nas funções pulmonares, renais, acúmulo de líquido no espaço intersticial, febre, leucocitose, vasoconstrição e maior susceptibilidade a infecções (MORAES et al., 2001).

Em relação aos achados hemodinâmicos, os resultados observados nos experimentos de TAMESUE et al. (2006) mostram queda da pressão arterial média. Porém, para INGYINN et al. (2004), a hipertensão é uma complicação comum durante e após o desvio, podendo ter muitos fatores como causas, tais como retenção à administração inadvertida de fluidos durante o procedimento e estresses hormonais de adrenalina, noradrenalina e cortisol.

Por tais motivos, os sinais vitais necessitam ser acompanhados a todo o momento. Nesse contexto, a monitoração hemodinâmica invasiva torna-se essencial em conjunto com a ECMO, contribuindo com a avaliação da função cardíaca e volumétrica. A observação dos sinais vitais pode refletir com certo grau de segurança à função cardiovascular e à perfusão (GAY et al., 2005).
Ainda sobre a função cardiovascular, BALASUBRAMANIAN et al. (2007) descrevem algumas arritmias relacionadas à ECMO após a retirada do paciente do circuito. Já KHAN et al. (2007) sugerem que o uso da ECMO pode estar associado ao aumento do risco de sangramentos e infartos.

Segundo a literatura, os marcadores cardíacos têm sido empregados rotineiramente para a detecção dos infartos agudos do miocárdio (ADAMS et al., 2007). Desses marcadores, destaca-se a creatinoquinase (CK-NAC), que é uma enzima utilizada em medicina experimental para quantificar os danos do músculo cardíaco e da musculatura esquelética. Além disso, pode ser também um sensível indicador de danos musculares. Para alguns propósitos clínicos, muitos tipos de células contêm CK com alta especificidade no músculo esquelético (KANEKO et al., 1997). A elevação sérica dessa enzima ocorre em virtude de danos nas células musculares, resultado de um estresse físico (DUNCAN \& PRASSER, 1986). A outra enzima em questão é a CK-MB, uma isoenzima híbrida da CK, existente no músculo cardíaco (LOPES et al., 2005).

Em humanos, a dosagem da CK-MB vem sendo utilizada como principal método para confirmação ou exclusão de infarto agudo do miocárdio, e picos de CK-MB podem prever eventos cardíacos desfavoráveis em populações de alto risco. Porém, CK e CK-MB são pobres marcadores de danos no miocárdio em cães. A CK-MB é um marcador cardíaco menos específico que a troponina cardíaca para essa espécie (LOPES et al., 2005; SANTOS, 2005; DINIZ et al., 2007). Durante o evento de hipóxia e necrose de miocárdio, as enzimas são liberadas para a corrente sanguínea, aumentando consideravelmente sua concentração plasmática (SANTOS, 2005; DINIZ et al., 2007).

ETTINGER \& FELDMAN (1997) informam que, pelo fato de o infarto do miocárdio decorrente da oclusão de um ramo importante da artéria coronária ser evento raro no cão, a CK-MB não é uma enzima frequentemente solicitada na rotina veterinária, ficando restrita aos estudos experimentais.

A similaridade morfológica e fisiológica entre o coração dos cães e dos humanos faz com que os caninos sejam utilizados como modelos experimentais de afecções cardíacas para humanos. Entretanto, em relação à $\mathrm{CK}-\mathrm{MB}$, sua distribuição é diferente entre essas espécies, uma vez que, no humanos, a maior 
concentração dessa enzima ocorre no ventrículo esquerdo nos humanos e nos cães no ventrículo direito (SANTOS, 2005).

De qualquer forma, uma vez mensurada, é importante considerar os valores da CK-MB em relação à CK total, para se determinar se está realmente aumentada. Ou seja, a porcentagem de CK-MB em relação ao valor total de CK fornece um dado confiável quando se avalia uma possível lesão do miocárdio, pois valores aumentados de CK-MB acompanhados de aumento de CK podem ser resultantes de esforço físico, por exemplo. Porém, quando se observa aumento de CK-MB não acompanhado de aumento da $\mathrm{CK}$ total, a porcentagem dessa isoenzima fica elevada, comprovando-se lesão no músculo cardíaco (MELO et al., 2008).

Dessa forma, pelo fato de a ECMO representar um método altamente invasivo e não fisiológico, procedeu-se a esta pesquisa, a fim de ser observado o comportamento das enzimas CK e CK-MB durante um período de três horas de oxigenação extracorpórea por membrana, objetivando detectar possíveis alterações cardíacas. Ainda, espera-se que os resultados observados possam contribuir com o aperfeiçoamento das técnicas e melhorar as margens de sobrevida dos pacientes submetidos à ECMO.

\section{MATERIAL E MÉTODOS}

Para a presente pesquisa utilizaram-se cinco cães $\mathrm{SRD}$, sem restrição de sexo e idade, presuntivamente adultos jovens, com peso corporal entre dez e quinze quilos. Todos os animais foram cedidos pelo Centro de Controle de Zoonoses de Campos dos Goytacazes, RJ.

Os animais foram admitidos no Hospital Veterinário, onde se realizaram avaliações clínicas, laboratoriais (hemograma e bioquímica sérica) e complementares (eletrocardiografia convencional e ecocardiografia em modo B e M), para a detecção de quaisquer alterações orgânicas. Os animais enfermos foram descartados do experimento.

Posteriormemte, alocaram-se os animais em gaiolas apropriadas, sendo fornecida alimentação especializada, água ad libitum. Para seu bem-estar, procedia-se à limpeza dos animais e a banhos de sol.

Os animais foram anestesiados seguindo o mesmo protocolo, constituído de $0,1 \mathrm{mg} \cdot \mathrm{kg}^{-1}$ de aceproma- zina ${ }^{1}$ por via intravenosa (IV), seguido de $2 \mathrm{mg} \cdot \mathrm{kg}^{-1} \mathrm{de}$ cetamina $^{2} \mathrm{IV}$, posteriormente $7 \mathrm{mg} \cdot \mathrm{kg}^{-1}$ de lidocaína ${ }^{3}$ por via epidural. Fez-se a manutenção anestésica com isoflurano $42,5 \%$.

A anticoagulação foi realizada com heparina ${ }^{5}$, na dose única de $4 \mathrm{mg} . \mathrm{kg}^{-1} \mathrm{IV}$.

Depois de anestesiados e entubados, os animais foram colocados em decúbito dorsal e submetidos à ECMO por meio da utilização da técnica de desvio arteriovenoso, com a retirada do sangue pela artéria femoral e a reinfusão pela veia femoral. Esse sangue seguia o seguinte trajeto: artéria femoral, tubo conector, oxigenador de membranas, e, após a remoção do $\mathrm{CO}_{2}$ e o recebimento de $\mathrm{O}_{2}$, o sangue fluía de volta para o organismo através de outro tubo conector, chegando à veia femoral. Os animais permaneceram sob ECMO durante 180 minutos, com o oxigenador de membranas "MASTERFLO infant” (Dideco®, Itália).

Nos animais submetidos à oxigenação extracorpórea por membrana, utilizou-se, ainda, um suporte ventilatório mínimo, com pressão positiva no final da expiração (PEEP) de 10 cm $\mathrm{H}_{2} \mathrm{O}$ e $\mathrm{FiO}_{2}$ de $21 \%$, concomitantemente à terapia de suporte promovida pela ECMO, objetivando evitar atelectasias, e as funções dos pulmões sendo substituídas apenas parcialmente pelo órgão artificial. Nesses animais também foi administrada dopamina ${ }^{6}$, na dose de $5 \mu \mathrm{g} / \mathrm{kg} /$ minuto, em infusão contínua, como suporte pressórico e inotrópico.

As amostras de sangue venoso foram coletadas por seringas de $3 \mathrm{ml}$ conectadas a um cateter longo localizado na veia jugular, o qual foi guiado em direção ao átrio direito. $\mathrm{O}$ sangue coletado foi repassado em frascos siliconizados sem anticoagulante. Estipulou-se em trinta minutos o intervalo entre as coletas durante três horas, totalizando sete amostras por animal durante a ECMO. Também se realizou a coleta de uma amostra de sangue do animal antes da ECMO, para controle,

1 Acepran - Univet S.A., Rua Clímaco Barbosa, 700 - Cambuci, São Paulo, SP.

2 Ketamina - Agener União Química Farmacêutica Nacional. Av. dos Bandeirantes, Planalto Paulista, São Paulo, SP.

3 Lidovet - Laboratório Bravet Ltda. Rua Visconde de Santa Cruz, 276 - Engenho Novo, Rio de Janeiro, RJ.

4 Isoforine - Cristália Produtos Químicos Farmacêuticos Ltda., Rod. Itapira-Lindóia, Km 14, Itapira, SP.

5 Heparin - Cristália Produtos Químicos Farmacêuticos Ltda., Rod. Itapira-Lindóia, Km 14, Itapira, SP.

6 Cloridrato de Dopamina - Laboratório Teuto Brasileiro S.A., VP 7-D módulo 11 - Quadra 13 - DAIA, Anápolis, GO. 
totalizando oito tempos de coleta durante o período total de experimentação.

As amostras sanguíneas foram centrifugadas (1.500 g por cinco minutos) em tempo não superior a uma hora, e o produto sobrenadante foi aliquotado após a coleta em volume mínimo de $500 \mu \mathrm{L}$. Acondicionaramse tais amostras em microtubos tipo "Eppendorf", sendo mantidas sob refrigeração em refrigerados, à temperatura de $-20^{\circ} \mathrm{C}$, para posterior realização de análises bioquímicas dos itens CK-Total e CK-MB, em equipamento espectrofotométrico Labmax Plenno, da marca Labtest $\AA$, utilizando-se kits comerciais da mesma marca.

Após o estudo, os animais foram submetidos à eutanásia com administração de tiopental sódico, na dose de 40mg. $\mathrm{kg}^{-1} \mathrm{IV}$, e cloreto de potássio, na dose de $100 \mathrm{mg} . \mathrm{kg}^{-1} \mathrm{IV}$, respeitando-se princípios éticos do uso de animais de experimentação. O presente trabalho foi licenciado pelo Comitê de Ética para Uso de Animais da Universidade Estadual do Norte Fluminense Darcy Ribeiro, sob o registro CEUA-UENF 038/2008.

Anotaram-se os resultados em fichas individuais (oito para cada animal), sendo as diferenças entre os momentos analisadas com programa estatístico GraphPad Prism versão 4.03 for Windows (GraphPad Software) através dos testes One-way ANOVA (Friedman e Kruskal-Wallis) com pós-teste de comparações múltiplas de Tukey, $\operatorname{com} \alpha=5 \%$.

Entre as variáveis foi aplicado o teste de Correlação de Pearson, $\operatorname{com} \alpha=5 \%$.

\section{RESULTADOS E DISCUSSAO}

\section{Creatino quinase (CK)}

A Figura 1 apresenta o comportamento da enzima creatino quinase (CK) dos animais submetidos à ECMO. Pela análise da linha de tendência apresentada, conclui-se que a CK apresentou uma tendência de aumento ao longo das três horas de ensaio. Por meio da análise dos valores médios obtidos em cada momento de avaliação, pode-se perceber que ocorreu um aumento estatisticamente significativo dos valores da CK ao longo das três horas de ECMO. Os valores são apresentados na Tabela 1.

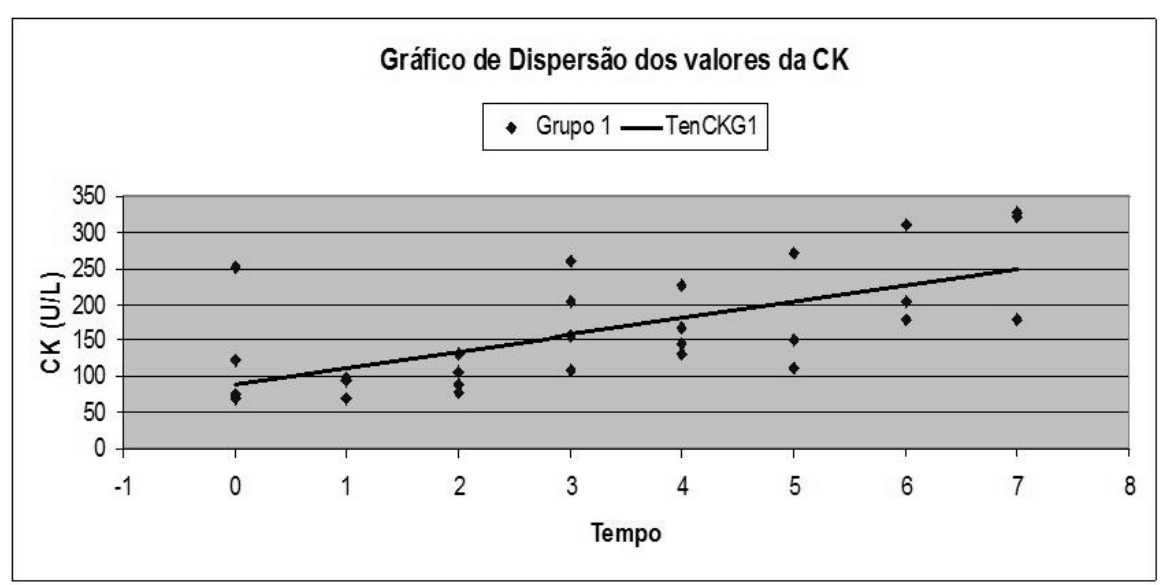

FIGURA 1. Gráfico de dispersão e comportamento da enzima creatino quinase (CK), em U/L, dos animais durante o período de três horas de ECMO. Hospital Veterinário/UENF, 2008.

TABELA 1. Médias e análise estatística do comportamento da enzima creatino quinase (CK) dos animais durante o período de três horas de ECMO. Hospital Veterinário/UENF, 2008.

\begin{tabular}{|c|c|c|c|c|c|c|c|c|}
\hline Tempos & M0 & M1 & M2 & M3 & M4 & M5 & M6 & M7 \\
\hline $\mathrm{CK}(\mathrm{U} / \mathrm{L})$ & 130,3 & 89,25 & 101,8 & 182,8 & 168,5 & 178,3 & 232,0 & 276,3 \\
\hline Análise estatística & \multicolumn{8}{|c|}{ ANOVA, significativo $\alpha=5 \%$; Tukey Signficativo $\alpha=5 \%$} \\
\hline Referência* (U/L) & \multicolumn{8}{|c|}{$20,0-220,0$} \\
\hline
\end{tabular}

* Referência segundo KANEKO et al., 1997. 
Dessa forma, pode ser observado que ocorreu uma alteração não apenas estatisticamente significativa no comportamento da CK durante a ECMO, mas também uma alteração fisiológica, visto que, de acordo com LOPES et al. (2005), o aumento da CK, além dos valores de normalidade, pode estar correlacionado a uma lesão muscular. Ainda segundo os autores, a CK é um indicador sensível e específico de lesão muscular, o que torna confiável a caracterização do dano muscular observado durante a ECMO.

Essa caracterização do dano muscular também é proposta por SOUZA \& ELIAS (2006). Esses autores afirmam que, durante a circulação extracorpórea, a distribuição dos fluxos de sangue para os diversos órgãos se altera e é diferente da distribuição normal, regulada pelo próprio organismo. Diante disso, os órgãos mais nobres, como o coração e o cérebro, recebem fluxos de sangue adequados, enquanto os rins, o fígado e, sobretudo, as grandes massas musculares recebem fluxos de sangue insuficientes para as suas necessidades. Em função desse baixo fluxo de perfusão, produz-se um quadro de hipóxia tecidual que favorece o dano muscular, caracterizado pelo aumento da CK no presente trabalho.

Creatino quinase MB (CK-MB)

A Figura 2 apresenta o comportamento da enzima creatino quinase $\mathrm{MB}(\mathrm{CK}-\mathrm{MB})$ dos animais do experimento submetidos à ECMO. Na figura, a análise da linha de tendência apresentada permite concluir que a CK-MB apresentou uma tendência de aumento ao longo das três horas de ensaio. Os valores reais médios para cada momento são descritos na Tabela 2.

A análise estatística empregada (Anova $(\alpha=5 \%))$ não permitiu concluir uma variação estatisticamente significativa ao longo das três horas de procedimento.

De acordo com SHARKEY et al. (1991), SANTOS (2005) e DINIZ et al. (2007), a CK-MB é uma isoenzima liberada para o meio extracelular quando há necrose de miocárdio, sendo um recurso importante para a detecção de lesão cardíaca. Logo, em cães, seu aumento pode ocorrer quando há um comprometimento do miocárdio secundário a diversas situações (como parvovirose, endocardite, dirofilariose e cardiomiopatia dilatada).

Entretanto, o comportamento de tendência de elevação da CK-MB do restante dos animais durante a ECMO não pode ser relacionado diretamente a tal comprometimento miocárdico, uma vez que, dentre outros motivos, a CK-MB também se encontra presente, em menor quantidade, em outros tecidos, o que reduz um pouco sua especificidade. Da mesma forma, os resultados de outras variáveis apresentadas neste estudo corroboram tal conclusão, como exposto a seguir.

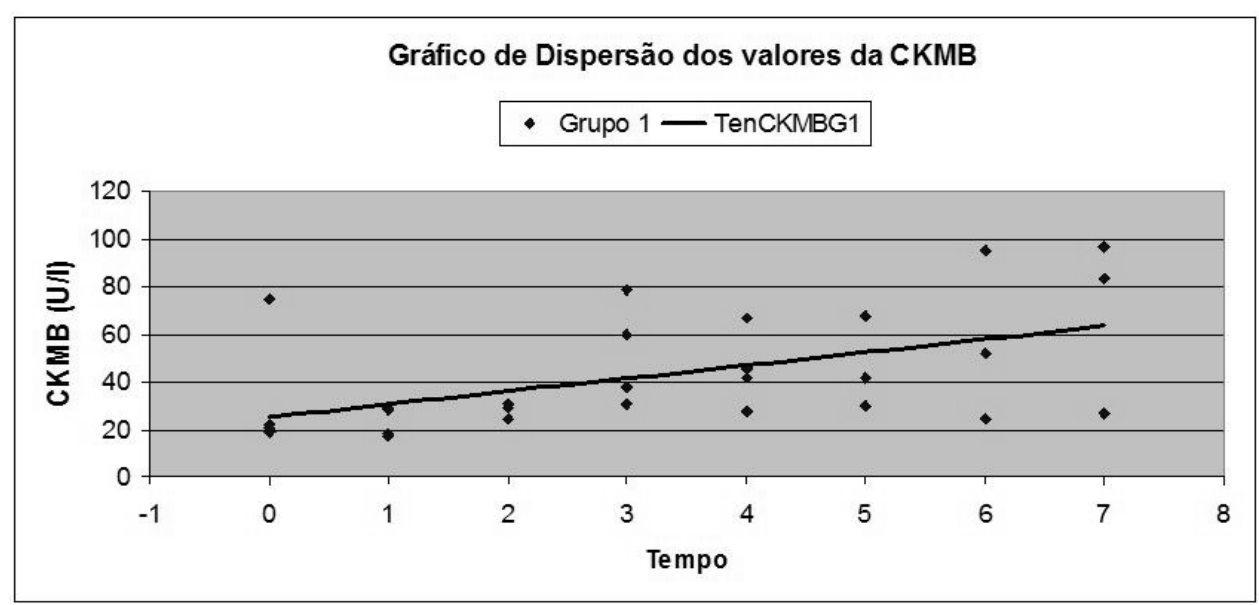

FIGURA 2. Gráfico de dispersão e comportamento da enzima creatino quinase MB (CK-MB), em U/L, dos animais durante o período de três horas de ECMO. Hospital Veterinário/UENF, 2008. 
TABELA 2. Médias e análise estatística do comportamento da enzima creatino quinase MB (CK-MB) durante o período de três horas de ECMO. Hospital Veterinário/UENF, 2008.

\begin{tabular}{lcccccccc}
\hline Tempos & M0 & M1 & M2 & M3 & M4 & M5 & M6 & M7 \\
\hline CK-MB (U/L) & 33,90 & 23,18 & 26,90 & 51,55 & 45,24 & 45,95 & 56,89 & 68,63 \\
Análise estatística & \multicolumn{7}{c}{ ANOVA, não significativo $\alpha=5 \%$} \\
Referência* (U/L) & \multicolumn{8}{c}{$17,07-30,73$} \\
\hline
\end{tabular}

Referência segundo LOPES et al., 2005.

\section{Relação CK versus CK-MB}

Segundo MELO et al. (2008), embora a CK-MB possa ser considerada um marcador de lesão miocárdica, esta enzima não deve ser avaliada de forma isolada. Para o autor, é importante considerar os valores de CK-MB em relação à CK total para se determinar se a primeira está realmente aumentada. Dessa maneira, um cálculo de porcentagem de CK-MB em relação ao valor total de CK fornece um dado mais confiável quando se avalia uma possível lesão do miocárdio, uma vez que valores aumentados de CK-MB acompanhados de aumento de CK podem ser resultantes de uma lesão muscular em geral.

Porém, quando se observa aumento de CK-MB não acompanhado de aumento da $\mathrm{CK}$ total, a porcentagem dessa isoenzima fica elevada, comprovando-se lesão no músculo cardíaco (MELO et al., 2008).

Embora não exista um parâmetro de normalidade para o percentual de CK-MB em função do valor total de CK em cães, a Figura 3 apresenta o comportamento dessas duas enzimas ao longo do período a que os animais foram submetidos à ECMO. De acordo com SHARKEY et al. (1991) e MELO et al. (2008), a título de comparação, na espécie humana, esses valores situam-se entre $14 \%$ e $25 \%$ da CK total.

Como pode ser observado, o comportamento das duas enzimas é semelhante ao longo de todo o experimento, e a Tabela 3 mostra que as porcentagens de CK-MB em relação a CK em todos os momentos avaliados foram semelhantes.

A Tabela 3 demonstra, ainda, que, através de uma análise estatística por meio da Correlação de Pearson " $r$ " $(\alpha=5 \%)$, o comportamento das duas enzimas foi similar durante a ECMO, pois apresentou $\mathrm{r}=$ 0,9908 . Dessa forma, pode-se concluir que o aumento da CK-MB durante o experimento deveu-se, propriamente, ao aumento da CK. Por extensão, infere-se que, neste caso, a CK-MB aumentou em função de um dano muscular geral, e não miocárdico.

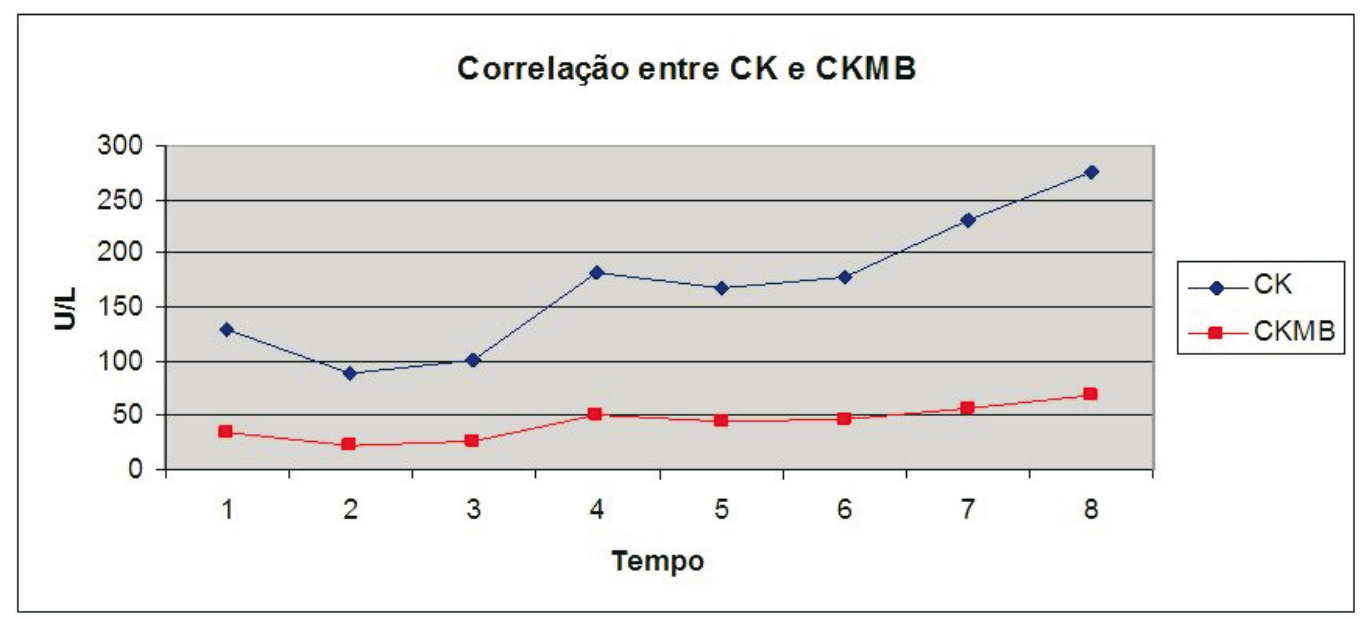

FIGURA 3. Gráfico do comportamento da enzima creatino quinase $M B(C K-M B)$, em U/L, em função da enzima creatino quinase (CK) dos animais durante o período de três horas de ECMO. Hospital Veterinário/UENF, 2008. 
TABELA 3. Médias e análise estatística do comportamento da enzima creatino quinase MB (CK-MB) em função da creatino quinase (CK) durante o período de três horas de ECMO. Hospital Veterinário/UENF, 2008.

\begin{tabular}{lcccccccc}
\hline Tempos & M0 & M1 & M2 & M3 & M4 & M5 & M6 & M7 \\
\hline CK (U/L) & 130,3 & 89,25 & 101,8 & 182,8 & 168,5 & 178,3 & 232,0 & 276,3 \\
CK-MB (U/L) & 33,90 & 23,18 & 26,90 & 51,55 & 45,24 & 45,95 & 56,89 & 68,63 \\
Correlação Pearson $\alpha=5 \%$ & & \multicolumn{7}{c}{$r=0,9908$} \\
\% CK-MB em CK & 26,02 & 25,98 & 26,43 & 28,21 & 26,85 & 25,76 & 24,52 & 24,83 \\
Referência (U/L) & & \multicolumn{7}{c}{$17,07-30,73$} \\
\hline
\end{tabular}

\section{CONCLUSÕES}

Diante os resultados apresentados e de seu confrontamento com a literatura disponível, pode-se concluir que a ECMO induziu uma lesão muscular evidente, sobretudo em tecidos periféricos, representada pelos aumentos séricos de CK e CK-MB. Entretanto, com base na mesma literatura, não se permite concluir que a ECMO tenha induzido lesões isquêmicas de miocárdio durante o período de três horas, uma vez que o aumento da CK-MB não ocorreu de forma isolada, e sim em fator de forte correlação com a CK.

\section{AGRADECIMENTOS}

Ao laboratório Terceiro Milênio (Pedra Verde), pela disponibilização do equipamento espectrofotômétrico para realização dos ensaios bioquímicos.

\section{REFERÊNCIAS}

ADAMS, J. E.; APPLE, F.; JAFFE, A. S. Markers in cardiology: a case-oriented approach. Massachusetts: Blackwell Publishing, 2007. 268 p.

BALASUBRAMANIAN, S. K.; TIRUVOIPATI, R.; AMIN, M.; AABIDEEN, K. K.; PEEK, G. J.; SOSNOWSKI, A. W.; FIRMIN, R. K. Factors influencing the outcome of paediatric cardiac surgical patients during extracorporeal circulatory support. Journal of Cardiothoracic Surgery, v. 2, n. 4, p. 1-9, 2007.

BARTLETT, R. H.; FONG, S. W.; BURNS, N. E.; GAZZANIGA, A. B. Prolonged partial venoarterial bypass: physiologic, biochemical, and hematologic responses. Annals of Surgery, v. 180, n. 6, p. 850-856, 1974.

DINIZ, P. P. V. P.; SCHWARTZ, D. S.; COLLICCHIO-ZUANAZE, R. C. Cardiac trauma confirmed by cardiac markers in dogs: two case reports. Arquivo Brasileiro de Medicina Veterinária e Zootecnia, v. 59, n. 1, p. 85-89, 2007.

DUNCAN, J. R.; PRASSER, K. W. Veterinary laboratory medicine. Iowa: State University Press, 1986. 285 p.

ETTINGER, S. J.; FELDMAN, E. C. Tratado de medicina interna veterinária de pequenos animais: moléstias do cão e do gato. São Paulo: Manole, 1997. 3020 p.

GAY, S. E.; ANKNEY, N.; COCHRAN, J. B.; HIGHLAND, K. B. Critical Care Challenges in the Adult ECMO Patient. Dimensions of Critical Care Nursing, v. 24, n. 4, p. 157-162, 2005.

INGYINN, M.; RAIS-BAHRAMI, K.; EVANGELISTA, R.; HOGAN, I.; RIVERA, O.; MIKESELL, G. T.; SHORT, B. L. Comparison of the effect of venovenous versus venoarterial extracorporeal membrane oxygenation on renal blood flow in newborn lambs. Perfusion, v. 19, p. 163-170, 2004.

KAHN, J.; MULLER, H.; MARTE, W.; REHAK, P.; WASLER, A.; PRENNER, G.; TSCHELIESSNIGG, K. Establishing extracorporeal membrane oxygenation in a university clinic: case series. Journal of Cardiothoracic and Vascular Anesthesia, v. 21, n. 3, p. 384-387, 2007.

KHAN, N. U.; AL-ALOUL, M.; KHASATI, N.; MACHAAL, A.; LEONARD, C. T.; YONAN, N. Case report: extracorporeal membrane oxygenator as a bridge to successful surgical repair of bronchopleural fistula following bilateral sequential lung transplantation: a case report and review of literature. Journal of Cardiothoracic Surgery, v. 2, n. 28, p. 1-6, 2007.

KANEKO, J. J.; HARVEY, J. W.; BRUSS, M. L. Clinical biochemestry of domestic animals. Califórnia: Academic Press, 1997. 932 p.

LIN, C.; CHEN, Y.; TSAI, F.; TIAN, Y.; JENQ, C.; FANG, J.; YANG, C. RIFLE Classification is predictive of short-term prognosis in critically Ill patients with acute renal failure supported by extracorporeal membrane oxygenation. Nephrology Dialysis Transplantation, v. 21, p. 2867-2873, 2006. 
LOPES, S. T. A.; FRANCISCATO, C.; TEIXEIRA, L. V.; OLIVEIRA, T. G. M.; GARMATZ, B. C.; VEIGA, A. P. M.; MAZZANTI, A. Determinação da creatina quinase em cães. Revista da FZVA Uruguaiana, v. 12, n. 1, p. 116-122, 2005.

MELO, M. M.; VERÇOSA JÚNIOR, D.; PINTO, M. C. L.;SILVEIRA, J. B.; FERRAZ, V.; ECCO, R. PAES, P. R. O. Intoxicação experimental com extratos de Mascagnia rigida (Malpighiaceae) em camundongos. Arquivo Brasileiro de Medicina Veterinária e Zootecnia, v. 60, n. 3, p. 631-640, 2008.

MORAES, M. C. J.; MORAES, D. J.; BASTOS, E. S.; MURAD, H. Circulação extracorpórea com desvio veno-arterial e baixa pressão parcial de oxigênio. Revista Brasileira de Cirurgia Cardiovascular, v. 16, n. 3, p. 251-261, 2001.

MOSCARDINI, A. C.; GODOY, M. F.; BRAILE, D. M.; GODOY, J. M. P.; SOARES. M. J.; BRANDI, A. C.; RAMIN, S. L. Oxigenação extracorpórea por membrana e alterações hematológicas em estudo experimental. Revista Brasileira de Hematologia e Hemoterapia, v. 24, n. 2, p. 97-104, 2002.

PEEK, G. J.; CLEMENS, F.; ELBOURNE, D.; FIRMIN, R. K.; HARDY, P.; HIBBERT, C.; KILLER, H.; MUGFORD, M.; THALANANY, M.; TIRUVOIPATI, R.; TRUESDALE, A.; WILSON, A. CESAR: conventional ventilatory support vs extracorporeal membrane oxygenation for severe adult respiratory failure. BMC Health Services Research, v. 6, n. 163, p. 1-13, 2006.

SANTOS, A. L. F. Dosagem sérica da enzima creatinafosfoquinase-isoenzima MB (CK-MB) e de troponina I (cTnI) de cães eletrocardiograficamente normais e naqueles com desnível (infra e supra) do segmento ST, utilizando ensaio imunométrico por quimioluminescência. São Paulo: Universidade de
São Paulo (USP), 2005. 62 f. Disponível em: http://www.teses. usp.br/teses/disponiveis/10/10136/tde-09042008-131819. Acesso em: 18 mar. 2009.

SOUZA, M. H. L..; ELIAS, D. O. Fundamentos da circulação extracorpórea. Rio de Janeiro: Centro Editorial Alfa, 2006. 809 p.

TAMESUE, K.; ICHIBA, S.; NAWA, S.; SHIMIZU, N. An experimental study on pumpless extracorporeal membrane oxigenation (Ecmo) support in a canine model. Acta Medica Okayama, v. 60, n. 3, p. 167-172, 2006.

THAKAR, D. R.; SINHA, A. C.; WENKER, O. C. Concepts of neonatal ECMO. The Internet Journal of Anesthesiology, v. 5, n. 3, 2001. Disponível em: <http://www.ispub.com/ostia/index. php?xmlFilePath=journals/ija/vol5n3/ecmo.xml>.

Acesso em: 18 jan. 2008.

THAKAR, D. R.; SINHA, A. C.; WENKER, O. C. Conceitos sobre ECMO neonatal. Revista Latinoamericana de Tecnologia Extracorporea, v. 9, n. 1, 2000. Disponível em: <http://perfline.com/ revista/volume9/v9n1/v9n1-01.html>. Acesso em: 18 jan. 2008.

SHARKEY, S. W.; MURAKAMI, M.; SMITH, S. A.; APPLE, F. Canine myocardial creatine kinase isoenzyme response to coronary artery occlusion. Circulation, v. 84, p. 333-340, 1991.

WEBER, T. R.; CONNORS, R. H.; TRACY JR, T. F.; BAILEY, P. V.; STEPHENS, C.; KEENAN, W. Prognostic determinants in extracorporeal membrane oxygenation for respiratory failure in newborns. The Annals of Thoracic Surgery, v. 50, p. 720-723, 1990.

ZWISCHENBERGER, J. B.; STEINHORN, R. H.; BARTLETT, R. H. ECMO. Extracorporeal cardiopulmonary support in critical care. Michigan: Extracorporeal Life Support Organization, 2000. 822 p.

Protocolado em: 8 maio 2009. Aceito em: 18 jan. 2010. 